\title{
Cluster structures within Fermionic Molecular Dynamics
}

\author{
T. Neff, H. Feldmeier
}

Gesellschaft für Schwerionenforschung mbH, Planckstraße 1, 64291 Darmstadt, Germany

The many-body states in an extended Fermionic Molecular Dynamics approach are flexible enough to allow the description of nuclei with shell model nature as well as nuclei with cluster and halo structures. Different many-body configurations are obtained by minimizing the energy under constraints on collective variables like radius, dipole, quadrupole and octupole deformations. In the sense of the Generator Coordinate Method we perform variation after projection and multiconfiguration calculations. The same effective interaction derived from realistic interactions by means of the Unitary Correlation Operator Method is used for all nuclei. Aspects of the shell model and cluster nature of the ground and excited states of ${ }^{12} \mathrm{C}$ are discussed. To understand energies and radii of neutron-rich He isotopes the soft-dipole mode is found to be important.

\section{FERMIONIC MOLECULAR DYNAMICS}

In the Fermionic Molecular Dynamics (FMD) model [1] the A-body state is given as a Slater determinant $|Q\rangle$ of single-particle states $\left|q_{i}\right\rangle$

$|Q\rangle=\mathcal{A}\left\{\left|q_{1}\right\rangle \otimes \ldots \otimes\left|q_{A}\right\rangle\right\}$

The single-particle wave functions are described by Gaussian wave packets localized in phasespace

$\langle\boldsymbol{x} \mid q\rangle=\sum_{i} c_{i} \exp \left\{-\frac{\left(\boldsymbol{x}-\boldsymbol{b}_{i}\right)^{2}}{2 a_{i}}\right\}\left|\chi_{i}\right\rangle \otimes|\xi\rangle$.

In contrast to the AMD approach [2] in the FMD model the width parameter $a$ is treated as a complex variational parameter that can be different for each single-particle state. The spin orientation given by the spinor $|\chi\rangle$ is also treated as a variational parameter. For light nuclei the description can be improved by using a superposition of two Gaussian wave packets.

The FMD many-particle state is determined by minimizing the intrinsic energy

$E[|Q\rangle]=\frac{\left\langle Q\left|H_{e f f}-T_{c m}\right| Q\right\rangle}{\langle Q \mid Q\rangle}$

with respect to the parameters of all single-particle states. This corresponds to a Hartree-Fock calculation using the FMD single-particle basis. To improve upon this mean-field result we 
restore the symmetries of the Hamiltonian and perform a parity and angular momentum projection. This defines the projection after variation (PAV) result.

The effects of the projection can be quite large and a variation after projection calculation (VAP) should be done [ 2]. But as a full VAP calculation is very expensive we perform VAP calculations in the spirit of the Generator Coordinate Method. The intrinsic energy is minimized under constraints on collective coordinates like radius, dipole, quadrupole or octupole moments obtaining a set of many-body configurations. The VAP minimum is then obtained by minimizing the projected energies of these configurations as a function of the constraints. These configurations can then also be used in a multiconfiguration calculation (MC) where the projected Hamiltonian is diagonalized within the set of these configurations. This provides us with a well defined procedure to study the properties of the ground and the excited states.

\section{EFFECTIVE INTERACTION}

We use an effective interaction that is derived from the realistic Bonn or Argonne interactions by means of the Unitary Correlation Operator Method (UCOM) [ 3, 4]. The correlated interaction includes the short-range radial and tensor correlations induced by the repulsive core and the tensor force. The correlated interaction no longer connects to high momenta and can thus be used directly within product-state model spaces. The effects of three-body correlations and genuine three-body forces are at this stage simulated by an additional two-body force that contains momentum-dependence and spin-orbit forces. This correction term is adjusted to reproduce the binding energies and radii of ${ }^{4} \mathrm{He},{ }^{16} \mathrm{O},{ }^{40} \mathrm{Ca},{ }^{24} \mathrm{O}$ and ${ }^{48} \mathrm{Ca}$. Here we find for ${ }^{16} \mathrm{O}$ and ${ }^{40} \mathrm{Ca}$ that after angular momentum projection the tetrahedral configurations (see Figure 1 with $\alpha$-clusters are favored energetically versus the spherical shell model configurations by about $5 \mathrm{MeV}$. Altogether we need a $15 \%$ correction to the $a b$-initio correlated two-body interaction.
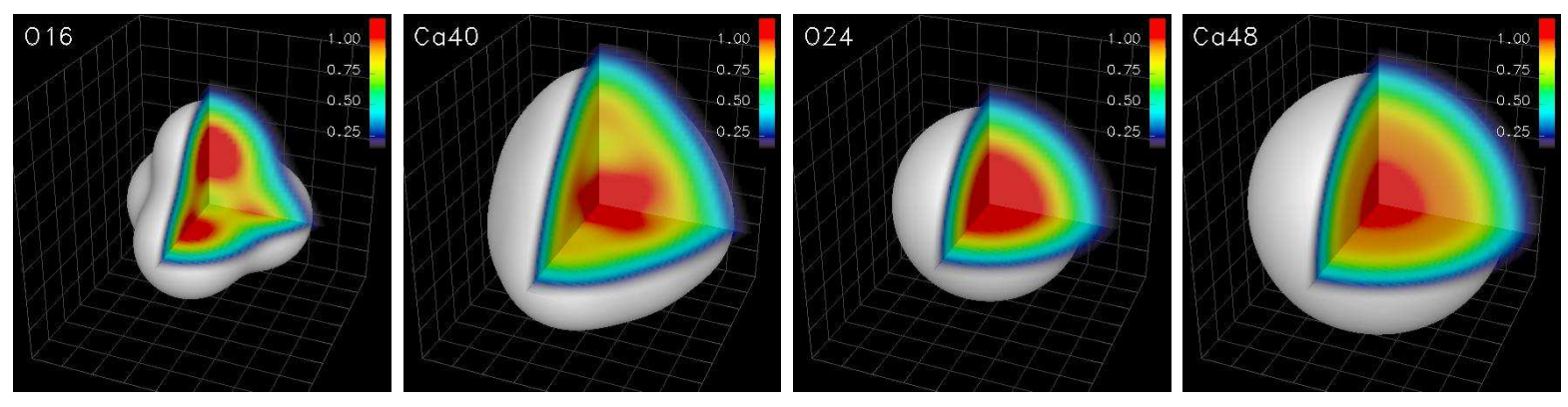

Figure 1. The effective interaction is fitted to tetrahedral ${ }^{16} \mathrm{O}$ and ${ }^{40} \mathrm{Ca}$ and spherical ${ }^{24} \mathrm{O}$ and ${ }^{48} \mathrm{Ca}$ configurations.

\section{GROUND AND EXCITED STATES OF ${ }^{12} \mathrm{C}$}

The question of the clustering in ${ }^{12} \mathrm{C}$ has a long history and has been studied in the AMD framework [ 5, 6]. Our effective interaction is fitted to binding energies and radii of ${ }^{4} \mathrm{He}$ and ${ }^{16} \mathrm{O}$ but has not been adjusted to ${ }^{12} \mathrm{C}$. We perform the FMD variation and obtain the $\left(1 s_{1 / 2}\right)^{4}\left(1 p_{3 / 2}\right)^{12}$ shell model configuration (V), displayed in the left part of Figure 2 . As it is spherical the angular momentum projection (PAV) does not affect the result. The binding energy obtained with this configuration is $7.5 \mathrm{MeV}$ too low compared to the experimental value (see Table 1). The 


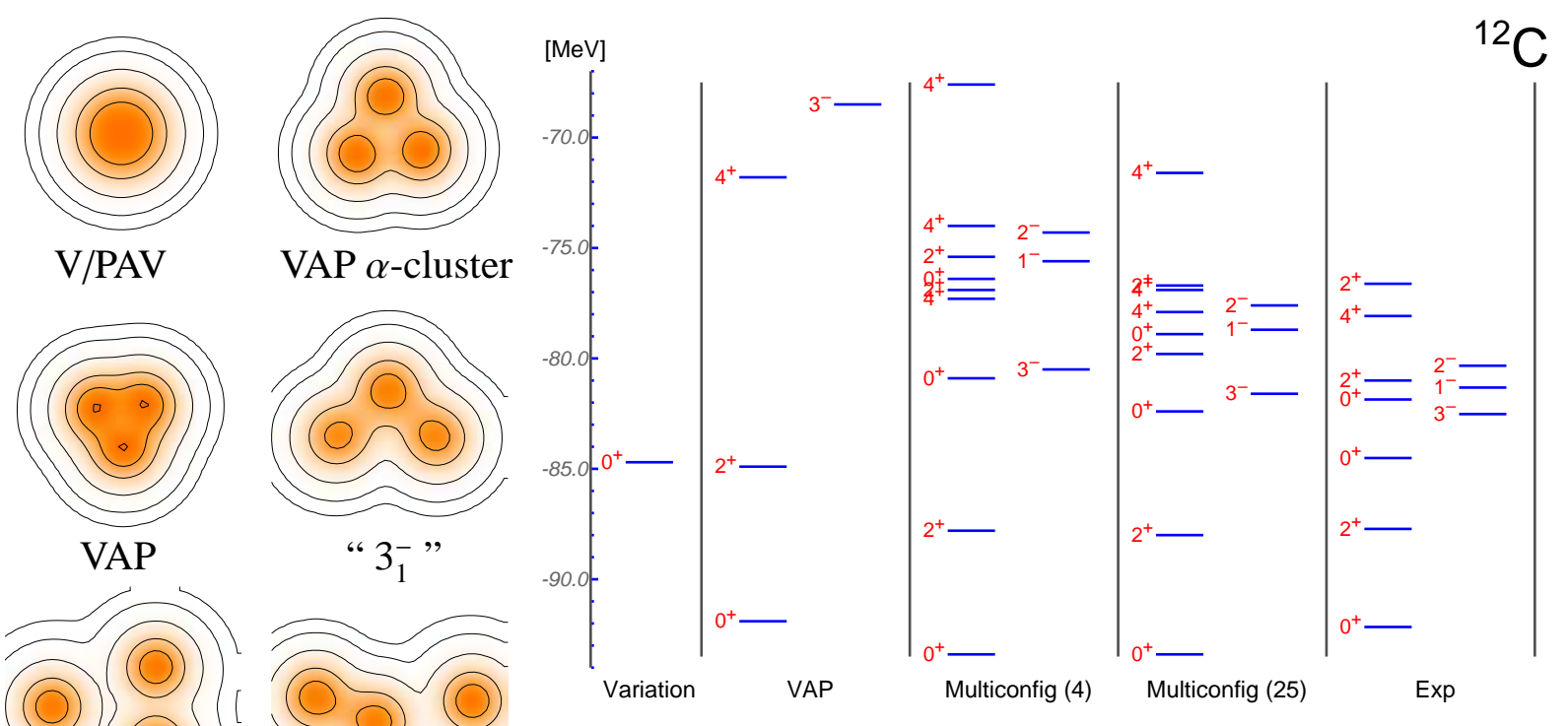

Figure 2. Intrinsic shapes used in the ${ }^{12} \mathrm{C}$ calculations (1.h.s.). " $\mathrm{O}_{2}^{+}$" “ $\mathrm{O}_{3}^{+} "$ Calculated and experimental spectrum of ${ }^{12} \mathrm{C}$ (r.h.s.)

obtained radius is too small. We can also restrict the intrinsic state to pure $\alpha$-cluster configurations. In these configurations the spin-orbit interaction does not contribute and already in the mean-field calculation a triangular configuration is preferred. In a VAP calculation where the positions and the widths of the $\alpha$ 's are the generator coordinates we obtain $4.3 \mathrm{MeV}$ less binding than with the shell model configuration and the radius is too large for the pure $\alpha$-cluster configuration. If we perform a variation after projection calculation with the radius and the octupole moment as generator coordinates we obtain the triangular configuration with polarized $\alpha$-clusters denoted by VAP on the 1.h.s. of Figure 2, It represents an intermediate between the VAP $\alpha$-cluster and the shell model (V/PAV) shape. The description of this polarization is strongly affected by using two Gaussians per single-particle state. We obtain $4.2 \mathrm{MeV}$ more binding compared to a VAP calculation with only one Gaussian per single-particle state. In case of the shell-model configuration the energy gain is only $1.5 \mathrm{MeV}$. The radius obtained in the VAP calculation is a little bit too small when compared to experiment. For the intrinsically deformed VAP configurations we can project out not only a $0^{+}$but also $2^{+}, 4^{+}$and $3^{-}$states (as

Table 1

Binding energies, charge radii and $B E(2)$-values of ${ }^{12} \mathrm{C}$.

\begin{tabular}{lrrr}
\hline & $E_{b}[\mathrm{MeV}]$ & $r_{\text {charge }}[\mathrm{fm}]$ & $B(E 2)\left[e^{2} \mathrm{fm}^{4}\right]$ \\
\hline V/PAV & 84.7 & 2.33 & - \\
VAP $\alpha$-cluster & 80.4 & 2.66 & 56.3 \\
VAP & 91.9 & 2.38 & 24.7 \\
Multiconfig(4) & 93.4 & 2.50 & 40.0 \\
\hline Exp & 92.2 & 2.47 & $39.7 \pm 3.3$ \\
\hline
\end{tabular}


shown in the VAP column of the spectrum in Figure 2) and can calculate the $B(E 2)$ value for the $0^{+} \rightarrow 2^{+}$transition using bare charges. The $B(E 2)$ value obtained for the VAP configuration is too small compared to the experimental value.

To improve the description we perform multiconfiguration calculations. For the additional configurations quadrupole and octupole moments are used as generator coordinates. In Figure 2 the configurations giving the biggest contributions for the first $3^{-}$and the second and third $0^{+}$states are shown. In a multiconfiguration calculation containing the VAP and these three intrinsic states the binding energy of the ground state is increased by $1.5 \mathrm{MeV}$. For the electromagnetic properties the effect of admixing these less compact configurations is more pronounced. The radius is increased and the $B(E 2)$ value is now in agreement with the experimental value. The spectrum obtained with these four intrinsic states is shown in the Multiconfig(4) column of Figure 2 Using 25 configurations the energies for the ground state and the lowest excited states change only slightly. For the higher excited states the effect is much bigger. Comparing to the experimental spectrum our results for the excited $0^{+}$and $2^{+}$states are already quite reasonable. Whereas the second $0^{+}$state seems to be mainly built of three $\alpha$ configurations, the third $0^{+}$ appears to be mainly ${ }^{8} \mathrm{Be}$ plus $\alpha$. A detailed study of these $0^{+}$and also $2^{+}$states will be done in the future. We expect that even more extended configurations will have to be considered, taking into account the proposed Bose condensed nature of these states around the three $\alpha$ threshold [ 7].

\section{HELIUM ISOTOPES}

To calculate the properties of the He isotopes we performed FMD calculations with two Gaussians per single-particle state using the electric dipole and the mass quadrupole moment as constraints for variation after projection (VAP) and multiconfiguration (Multiconf) calculations. In Table 2 the calculated binding energies and radii are shown together with the experimental values. In Figure 3 the intrinsic configurations corresponding to the VAP minima are displayed. With the two Gaussians the FMD wave function is able to describe the neutron halo around the $\alpha$-core. Using only a single Gaussian an imperfect halo together with a distorted $\alpha$-core is observed. Compared to the calculation using a single Gaussian the neutron-rich He isotopes

Table 2

Experimental and calculated binding energies and radii for the He isotopes.

\begin{tabular}{lllrrrr}
\hline & & ${ }^{4} \mathrm{He}$ & ${ }^{5} \mathrm{He}$ & ${ }^{6} \mathrm{He}$ & ${ }^{7} \mathrm{He}$ & ${ }^{8} \mathrm{He}$ \\
\hline PAV & $E_{b}[\mathrm{MeV}]$ & 28.3 & 25.4 & 26.3 & 26.5 & 29.8 \\
VAP & $E_{b}[\mathrm{MeV}]$ & 28.3 & 26.8 & 27.7 & 27.7 & 31.1 \\
Multiconf & $E_{b}[\mathrm{MeV}]$ & 28.4 & 27.4 & 29.1 & 28.7 & 31.7 \\
Exp & $E_{b}[\mathrm{MeV}]$ & 28.3 & 27.4 & 29.3 & 28.8 & 31.4 \\
\hline Multiconf & $r_{\text {mat }}[\mathrm{fm}]$ & 1.45 & 2.74 & 2.42 & 2.62 & 2.53 \\
Exp & $r_{\text {mat }}[\mathrm{fm}]$ & & & $2.48 \pm 0.03$ & & $2.52 \pm 0.03$ \\
\hline Multiconf & $r_{c h}[\mathrm{fm}]$ & 1.69 & 2.10 & 2.02 & 2.07 & 2.03 \\
Exp & $r_{c h}[\mathrm{fm}]$ & 1.68 & & & & \\
\hline
\end{tabular}



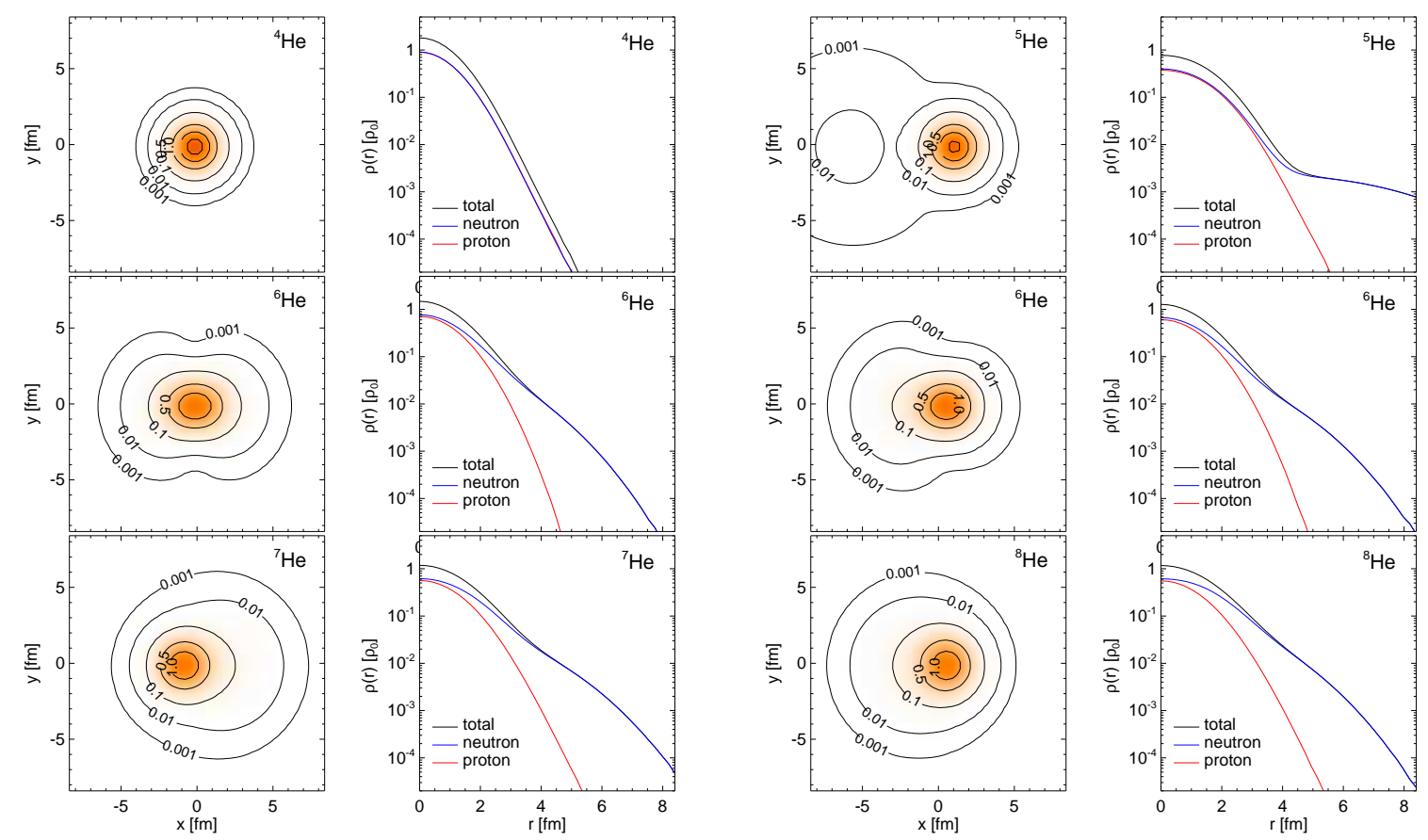

Figure 3. L.h.s.: intrinsic shapes corresponding to VAP minima. Cuts through nucleon density at $z=0$. R.h.s.: proton, neutron and nucleon densities as a function of radius.

gain about 3-5 MeV in the PAV case. For the ${ }^{6} \mathrm{He}$ nucleus we find a quadrupole deformed VAP minimum where the two halo neutrons are sitting on opposite sides of the core and a more bound (by about $1.1 \mathrm{MeV}$ ) dipole deformed VAP minimum where the neutrons are located on the same side of the core (see middle row of Figure 3). For the other isotopes we only find minima with respect to the dipole deformation. The multiconfiguration calculations that include shapes of different electric dipole moments reproduce the experimental staggering of the binding energies. The large measured matter radii of ${ }^{6} \mathrm{He}$ and ${ }^{8} \mathrm{He}$ are also well described by the multiconfiguration calculations. We also observe a significant increase in the charge radii of the neutron rich He isotopes compared to ${ }^{4} \mathrm{He}$. This is explained by the zero-point motion of the more or less unchanged $\alpha$-core with respect to the center of mass of the nucleus.

We conclude that the zero-point motion of paired neutrons in a soft-dipole mode seen in VAP and multiconfiguration mixing calculations is an essential ingredient for the understanding of neutron-rich $\mathrm{He}$ isotopes and probably also for other neutron halo nuclei.

\section{REFERENCES}

1. H. Feldmeier and J. Schnack, Rev. Mod. Phys. 72 (2000) 655. and references therein

2. Y. Kanada-En'yo and H. Horiuchi, Prog. Theor. Phys. Suppl. 142 (2001) 205

3. H. Feldmeier, T. Neff, R. Roth, J. Schnack, Nuc. Phys. A632 (1998) 61

4. T. Neff and H. Feldmeier, Nuc. Phys. A713 (2003) 311

5. Y. Kanada-En'yo, Phys. Rev. Lett. 81 (1998) 5291

6. N. Itagaki et al., Nuc. Phys. A719 (2003) 205c

7. Y. Funaki, A. Tohsaki, H. Horiuchi, P. Schuck, G. Röpke, Phys. Rev. C67 (2003) 051306 